\title{
Color Doppler Ultrasonography and Diffusion Weighted Magnetic Resonance Enterography in Pediatric Ibd: Comparison with Histology in the Evaluation of Disease Severity
}

\author{
Salinaro Emanuela ${ }^{*}$, Mancini Maria Elisabetta ${ }^{1}$, De Venuto Domenica², \\ Gallone Maria Filomena ${ }^{3}$, Russo Silvana ${ }^{4}$, Monteduro Mariantonietta ${ }^{1}$, \\ Angelelli Giuseppe ${ }^{1}$ \\ ${ }^{1}$ Interdisciplinary Department of Medicine, Section of Diagnostic Imaging, Aldo Moro University of Bari Medical \\ School, Bari, Italy \\ ${ }^{2}$ Department of Biomedicine of Developmental Age, Aldo Moro University of Bari, Bari, Italy \\ ${ }^{3}$ Department of Biomedical Science and Human Oncology, Aldo Moro University of Bari, Bari, Italy \\ ${ }^{4}$ Department of Emergency and Organ Transplantation, Pathology Division, Medical School, \\ Aldo Moro University of Bari, Bari, Italy \\ Email: ”emanuelasalinaro@gmail.com, elisabetta_mancini@hotmail.it,domenica.devenuto@policlinico.ba.it, \\ mfgallone@gmail.com, petrelli-@libero.it
}

Received 5 February 2016; accepted 20 February 2016; published 25 February 2016

Copyright (C) 2016 by authors and OALib.

This work is licensed under the Creative Commons Attribution International License (CC BY). http://creativecommons.org/licenses/by/4.0/

(c) (i) Open Access

\section{Abstract}

Objectives: The purpose of this study was to retrospectively compare color Doppler Ultrasonography (CD-US) and diffusion weighted Magnetic Resonance Enterography (DWI-MRE) in the evaluation of disease severity of pediatric inflammatory bowel diseases (IBD). Materials and methods: During the period between February 2010 and November 2012, 36 patients affected by IBD, aged between 7.8 and 18.5 years (mean 11.9 years) underwent, in the same week, CD-US, DWI-MRE [completed by apparent diffusion coefficient (ADC) calculation] and ileocolonoscopy with intestinal biopsies. Overall, 53 bowel segments ( 27 ileal, 26 colonic) were examined. At CD-US evaluation, a wall thickness $>3 \mathrm{~mm}$ in the small bowel and $>2 \mathrm{~mm}$ in the large bowel, associated with an increased intramural vascularity, were considered abnormal. ADC values were significant when lower than $2 \times 10^{-3} \mathrm{~mm}^{2} / \mathrm{sec}$. Histopathological grading of inflammation distinguished between active (mild, moderate and severe) and inactive disease. For each segment, CD-US findings and ADC values were compared with histology. Results: CD-US correctly graded 46/53 cases (86.8\%) as compared with histology. Statistical analysis demonstrated a negative correlation between ADC

\footnotetext{
*Corresponding author.
}

How to cite this paper: Emanuela, S., Elisabetta, M.M., Domenica, D.V., Filomena, G.M., Silvana, R., Mariantonietta, M. and Giuseppe, A. (2016) Color Doppler Ultrasonography and Diffusion Weighted Magnetic Resonance Enterography in Pediatric Ibd: Comparison with Histology in the Evaluation of Disease Severity. Open Access Library Journal, 3: e2406.

http://dx.doi.org/10.4236/oalib.1102406 
and histology, with the results corresponding in $43 / 53$ cases (81.1\%). Conclusions: Our study shows that both CD-US and DWI-MRE are non-invasive, useful diagnostic tools for the grading of IBD activity in children.

\author{
Keywords \\ Pediatric IBD, Crohn Disease, Ulcerative Colitis, Bowel Ultrasonography, Magnetic Resonance \\ Enterography, Disease Activity, Diffusion Weighted Imaging, Radiation Exposure
}

\author{
Subject Areas: Radiology \& Medical Imaging
}

\title{
1. Introduction
}

Inflammatory bowel diseases (IBD) are chronic, complex, multifactorial disorders which develop in genetically predisposed subjects [1]: they include Crohn's disease (CD), ulcerative colitis (UC) and indeterminate colitis (IC) [2] [3]. Several studies have reported that they are constantly on the increase [4] [5]: the prevalence of UC is 505 per 100,000 people in Europe and 249 per 100,000 in North America and of CD, 322 per 100,000 people in Europe and 319 per 100,000 in North America. Children and adolescents (aged between 0 and 18 years) account for about $25 \%$ - 30\% of the affected population [6]. The potential to cause lifelong ill health has an extremely high impact on the patient, their family or other carers and society [7]. Therefore, an early diagnosis and adequate follow-up timing are crucial, because they allow a proper management plan and therapy to be scheduled, avoiding unnecessary examinations [8].

Endoscopic and histological evaluation is the gold standard for the diagnosis and the grading of IBD, but it has some limitations [9]: it is an invasive procedure and it is often poorly tolerated, especially by the youngest patients. Moreover, it can be non-diagnostic in cases of significant stenosis.

Imaging modality plays a fundamental role in the diagnosis: it includes traditional radiology, ultrasonography (US), computerized tomography (CT) and magnetic resonance enterography (MRE).

Particularly, cross-sectional techniques (US, CT and MRE) have advanced the ability to diagnose, classify and monitor IBD [10]; their employment has radically modified the diagnostic approach coupling the study of the mucosal surface with a direct evaluation of wall thickening and extraluminal lesions.

Medical imaging in childhood must take into account the problem of dosimetry and radioprotection. This concept is strongly endorsed by the Society for Pediatric Radiology, particularly in the use of procedures and modalities involving higher radiation doses. Current imaging methods must be optimized for radiation dose reduction in pediatric patients who might be as much as ten times more radiosensitive than adults [11].

The use of trans-abdominal ultrasound for the assessment of patients with IBD is gaining popularity. The lack of ionizing radiation is particularly attractive when examining young patients undergoing frequent follow-up. However, even if US is widely available and relatively inexpensive [12], operator dependability and experience in the use for bowel assessment still pose major limitations [13].

MR is increasingly being used. Recent studies have assessed its ability to diagnose, to quantify disease extent, to detect inflammation or to show complications such as stenosis or fistula [14]. Its application is justified by the absence of ionizing radiation, along with very high soft-tissue contrast, multiplanar images, a low incidence of adverse events related to the use of intravenous contrast medium, and high diagnostic accuracy in the evaluation of luminal and extraluminal abnormalities [15].

Diffusion-weighted magnetic resonance (DWI-MR) imaging is a technique that relies on the diffusion of water in biologic tissues to produce images providing functional, quantitative information about tissue cellularity. The images are produced on the basis of the random (Brownian) motion of water molecules suspended in a liquid: each pixel is a representation of the average diffusion of water in the volume imaged [16]. Until now, no threshold of apparent diffusion coefficient (ADC), the quantitative parameter of DWI, has been established for defining inflammation in IBD [14].

At present, there is no single imaging modality that has been proven universally superior in either suspected or established IBD [17]. An important matter of concern, particularly in younger patients, is ionizing radiation 
exposure as a potential risk factor for cancer, especially considering that the risk of malignancies is increased in these patients due not only to the disease but also to treatments [18].

The aim of our study was to compare two non-ionizing techniques, namely color Doppler ultrasonography (CD-US) and diffusion weighted magnetic resonance enterography (DWI-MRE) with quantitative analysis of the ADC value, in the evaluation of the grade of activity in pediatric IBD, considering histology as the reference standard. To our knowledge, this study is the first to compare CD-US and ADC measurement for the definition of disease activity in pediatric IBD.

\section{Materials and Methods}

\subsection{Patients}

During the period between February 2010 and November 2012, 94 children and young adults (aged between 2.8 and 18.3 years, mean 11 years, median 13.15 years) underwent a pediatric gastroenterologic evaluation for suspected, non-diagnosed IBD or during follow-up for diagnosed IBD: CD-US and MRE were performed on this occasion in all the cases.

Among this group, we retrospectively selected all those subjects who had undergone, during the same week, CD-US, DWI-MRE with ADC calculation and intestinal biopsy: 36 patients met our inclusion criteria.

We excluded all patients aged more than 19 years, those who had not undergone one examination among US, MRE and histology, and those who did not undergo all three examinations during the same week.

The final sample consisted of 16 females (44.4\%) and 20 males (55.6\%), aged between 7.8 and 18.5 years (mean 11.9, median 12.9 years). Of these, 15 of 36 patients (41.7\%) were examined for suspected IBD, while 21 (58.3\%) had histological confirmation of the disease and were undergoing follow-up. Therefore, from 36 patients, 27 were affected by Crohn's disease, 4 by ulcerative colitis and 5 by indeterminate colitis. At the time of the observation, two patients had already undergone surgery and endoscopic dilatation for stricture complications.

\subsection{Imaging Modalities}

During the ultrasonographic and MR examination the bowel was considered in six distinct segments: terminal ileum, caecum, ascending colon, transverse colon, descending colon, sigmoid colon-rectum; overall, we examined 53 intestinal segments (27 ileal, 26 colonic).

\subsection{Ultrasound Examination}

Ultrasound examinations were performed by two experienced radiologists (G.A. and M.M. with 30 and 10 years of experience, respectively) blinded to the histology results, using a Philips HDI5000 SonoCT unit.

No special patient preparation was required prior to US other than a 6-h fasting without intake of solid foods and no contrast medium was used to fill the bowel.

After preliminary screening of the entire abdomen using a convex 2 - $5 \mathrm{MHz}$ probe, a linear 5 - $12 \mathrm{MHz}$ probe was used to study the small and large bowel loops (PRF 1000). Then the intramural vascularity was measured on color and power Doppler mode. The gain and the time gain compensation (TGC) were adjusted in order to minimize noise artifacts and to improve signal intensity. The parameters evaluated were bowel wall thickness (using a threshold of $3 \mathrm{~mm}$ for the terminal ileum and $2 \mathrm{~mm}$ for the colon) and intramural vascularity at the site of thickening. The severity of the disease was classified on the basis of the semiquantitative Limberg score, which couples wall thickening with the Doppler signal intensity. Grade 1 corresponds to a thickness greater than normal with partially obliterated wall layers but no increased vascularity. Grade 2 describes bowel wall thickening with spots of vascularity, while in grade 3 there are longer stretches of vascularity and in grade 4 the vascularized stretches extend into the mesentery [19] [20].

\subsection{MRE Protocol and Imaging Technique}

MRE examination was performed using a 1.5 Tesla scan (Philips, Achieva $1.5 \mathrm{~T}$ ), with phased array body coil (SENSE-body coil). Two experienced gastrointestinal radiologists (G.A. and M.M.), blinded to the histological findings, evaluated the images. 
All the patients were asked to stop eating solid foods for 3 days before MRE to cleanse the bowel. They also took oral cleansing laxatives (Movicol Norgine Italia Srl) for variable times and personalized doses. Allergic patients received anti-allergic premedication with corticosteroids and antihistamines.

On the day of the examination the patients drank a solution of biphasic contrast medium [polyethylene glycol (PEG)], previously prepared by dissolving a granular powder in $1000 \mathrm{ml}$ of water. The volume of contrast medium ranged from 200 and $1000 \mathrm{ml}$ depending on the patient's weight and was administered starting from one hour before MRE, in split doses and intervals based on the patient's compliance. No spasmolytic medication was used to inhibit bowel peristalsis: in all the cases a good degree of distension of the bowel loops was obtained. Patients were all studied in supine position.

The imaging protocol consisted of the following breath-hold sequences, acquired without administration of contrast medium: fluoroscopic rapid acquisition with relaxation enhancement (RARE), $120 \mathrm{~mm}$ thick, 10 seconds acquisition time, coronal and axial balanced fast field echo (B-FFE), coronal and axial T2w single shot turbo spin echo (SSh-TSE), axial T2w single shot turbo spin echo SPAIR (SSh-TSE-SPAIR), coronal and axial T1w high-resolution isotropic volume (THRIVE). After i.v. administration of $0.15 \mathrm{ml} / \mathrm{kg}$ of gadolinium diethylene-triamine penta acetic acid (Gd-DTPA) $0.5 \mathrm{M}$ followed by $20 \mathrm{ml}$ of saline solution, coronal and axial T1w high-resolution isotropic volume (THRIVE) sequences were performed. DWI sequences were acquired during free breathing, before the administration of Gd-DTPA, on the axial plane, setting b $0-600 \mathrm{~s} / \mathrm{mm}^{2}$, matrix $256 \times$ 256, slice number 40, thickness $7 \mathrm{~mm}$, TE $75 \mathrm{~ms}$, TR $8000 \mathrm{~ms}$, flip angle 90, FOV 350 - 450 (Table 1). DWI hyperintensity was considered abnormal and the ADC value was calculated at the same level on automatically generated ADC maps. Two small circular regions of interest (ROIs) with a diameter equivalent to the thickness of the intestinal wall were placed on each bowel segment assessed and the mean values were recorded for statistical analysis. The mean ROI area was $15 / 20 \mathrm{~mm}^{2}$.

\subsection{Histopathological Analysis}

A board-certified pathologist (S.R., with 20 years of experience in gastrointestinal pathology) performed the histological assessment on three ileal and/or colic samples per patient, obtained during ileocolonoscopy and stained with hematoxylin and eosin. Based on different grading scales for CD and UC, the disease was classified as inactive, mild, moderate or severe [21] [22]. Then, the CD-US and ADC results were compared with histological findings.

\subsection{Statistical Analysis}

Data on each patient, including demographic variables, CD-US results, ADC values and histological findings, were collected in a standardized form and then input into a database using File Maker Pro software and analyzed with STATA MP11 statistical software. CD-US and histological results were reported as frequency and percentage. ADC was indicated as mean value, with standard deviation (SD) and range. The agreement between CD-US and histology was calculated using Cohen's kappa coefficient. The results of each CD-US examination were compared with the histological grade of disease: sensitivity, specificity, positive predictive value (PPV) and negative predictive value (NPV) (95\% confidence interval) were calculated for each class of results. We

Table 1. Magnetic resonance imaging protocol.

\begin{tabular}{ll}
\hline \multicolumn{3}{c}{ Magnetic resonance protocol } \\
\hline 1 & Fluoroscopic rapid acquisition with relaxation enhancement (RARE) (120 mm thick, 10 seconds acquisition time) \\
2 & Coronal and axial balanced fast field echo (B-FFE) \\
3 & Coronal and axial T2wi single shot turbo spin echo (SSh-TSE) \\
4 & Axial T2wi single shot turbo spin echo SPAIR (SSh-TSE-SPAIR) \\
5 & Coronal and axial T1wi high-resolution isotropic volume (THRIVE) \\
6 & Axial DWI sequences with b 0 - 600 s/mm ${ }^{2}$ with autogenerated ADC map \\
7 & Coronal and axial T1wi high-resolution isotropic volume (THRIVE) after administration of 0.15 ml/kg of Gd-DTPA 0.5 M \\
\hline
\end{tabular}


also assessed the relation between ADC and histology using Spearman's correlation coefficient. For each test, 95\% Confidence Intervals (95\% CI) were indicated. Values of $\mathrm{p}<0.05$ were considered statistically significant. Informed written consent was obtained from all the patients aged 18 years or their legal guardians if younger than 18 years, for all diagnostic and therapeutic measures. The study was approved by the local ethics committee.

\section{Results}

The histological findings revealed inactivity in 4 out of 53 cases, mild activity in 19 cases, moderate activity in 18 and severe activity in 12 of 53 cases (Table 2).

Results of bowel CD-US (Figure 1) showed inactive disease in 5 of the 53 cases, mild active in 15 cases, moderate active in 19 cases and severe disease in 14 of the 53 cases (Table 3).

Therefore, in 46 out of the 53 cases (86.8\%) there was agreement between the ultrasonographic score and histological grading (4/4 inactivity, 15/19 mild activity, 18/18 moderate activity and 12/12 severe activity). In $5 / 53$ cases (9.4\%) CD-US overestimated the severity of the disease (mild histological activity vs moderate and severe US activity), while in 2/53 cases (3.8\%) there was an underestimation (moderate histological activity vs inactivity and mild US activity).

The observed agreement between CD-US and histology was 86.8\% (Table 4). Sensitivity, specificity, positive predictive value (PPV) and negative predictive value (NPV) for CD-US results, as compared to the histological classification of disease severity, are reported in Table 5.

All the abnormal intestinal segments showed hyperintense signal at DWI evaluation (Figure 2; Figure 3). The ADC values ranged from 0.17 to $1.986 \times 10^{-3} \mathrm{~mm}^{2} / \mathrm{sec}$. A negative correlation was found between ADC and histology (Spearman's coefficient -0.785 ; $\mathrm{p}<0.01$ ): the higher the ADC values $\left(>1 \times 10^{-3} \mathrm{~mm}^{2} / \mathrm{sec}\right.$ ), the lower the disease activity (inactive or mild). On the contrary, as disease activity increased, ADC tended to decrease $\left(<1 \times 10^{-3} \mathrm{~mm}^{2} / \mathrm{sec}\right)$, as shown in Figure 4 .

ADC results showed a correlation with the histological grade of activity in 43 out of 53 cases (81.1\%); no association was observed in the remaining $10(18.9 \%)$.

Assuming $1 \times 10^{-3} \mathrm{~mm}^{2} / \mathrm{sec}$ as the cut off value between active and inactive disease, we observed that values lower than $1 \times 10^{-3} \mathrm{~mm}^{2} / \mathrm{sec}$ corresponded to moderate-severe activity (31/37 cases, 83.8\%), while values higher than $1 \times 10^{-3} \mathrm{~mm}^{2} / \mathrm{sec}$ corresponded to inactivity or mild activity (12/16 cases, $75 \%$ ).

Table 2. Frequency distribution for histology results (with percentage and 95\% confidence interval indication).

\begin{tabular}{ccc}
\hline Histology results & $\mathbf{N}$ & $\mathbf{\%}$ \\
\hline Inactivity & 4 & $7.6(3.0-17.9)$ \\
Mild activity & 19 & $35.8(24.3-49.3)$ \\
Moderate activity & 18 & $34.0(22.7-47.4)$ \\
Severe activity & 12 & $22.6(13.4-35.5)$ \\
Total & $\mathbf{5 3}$ & $\mathbf{1 0 0 . 0}$ \\
\hline
\end{tabular}

Table 3. Frequency distribution for color Doppler ultrasonography results (with percentage and 95\% confidence interval indication).

\begin{tabular}{ccc}
\hline Ultrasonography results & $\mathbf{N}$ & \% \\
\hline Inactivity & 5 & $9.4(4.1-20.3)$ \\
Mild activity & 15 & $28.3(18.0-41.6)$ \\
Moderate activity & 19 & $35.9(18.0-41.6)$ \\
Severe activity & 14 & $26.4(16.4-39.6)$ \\
Total & $\mathbf{5 3}$ & $\mathbf{1 0 0 . 0}$ \\
\hline
\end{tabular}



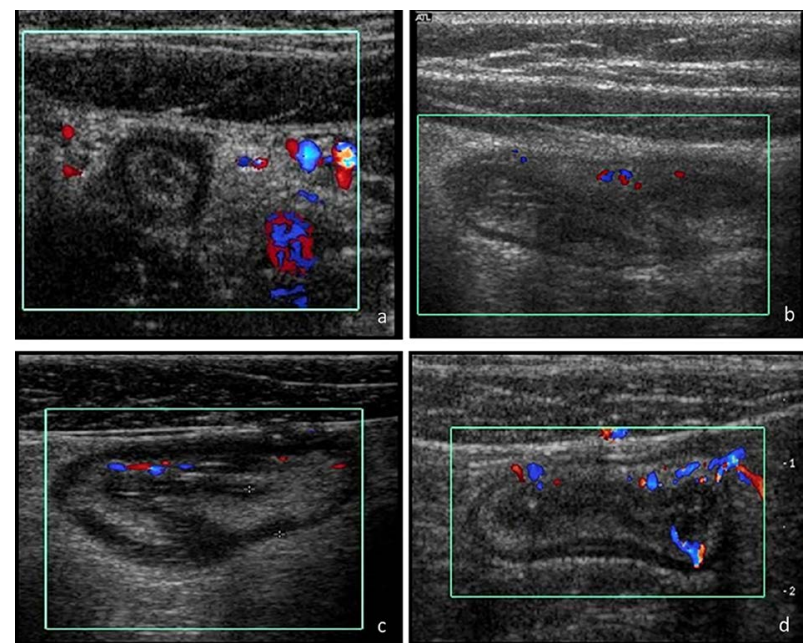

Figure 1. Color Doppler ultrasonography Limberg score. (a) Grade 1: Bowel wall thickening with partially obliterated wall layers, but no increased vascularity; (b) Grade 2: Bowel wall thickening with spots of vascularity; (c) Grade 3: Bowel wall thickening with longer stretches of vascularity; (d) Grade 4: Bowel wall thickening with stretches extending into the mesentery.
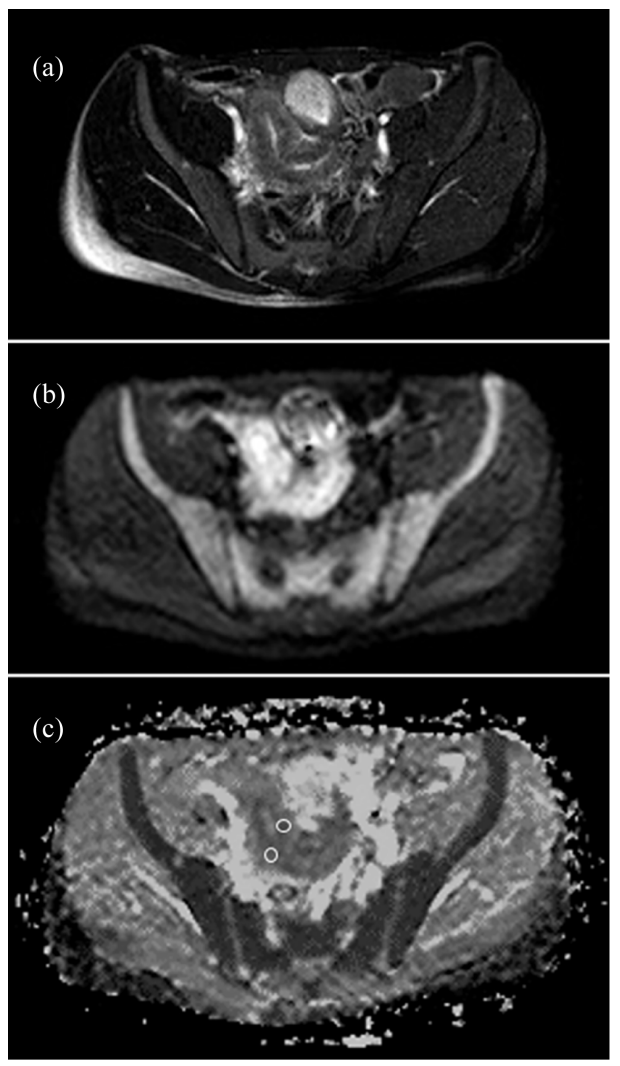

Figure 2. Crohn's disease. (a) Axial balanced fast field echo (B-FFE) sequence-marked circumferential terminal ileum bowel wall thickening; (b) Axial diffusion weighted (DWI) sequence-terminal ileum restricted diffusion with a hyperintense signal; (c) Apparent diffusion coefficient (ADC) map-the ADC value calculated at the same level was $1.326 \times 10^{-3}$ $\mathrm{mm}^{2} / \mathrm{s}$. 

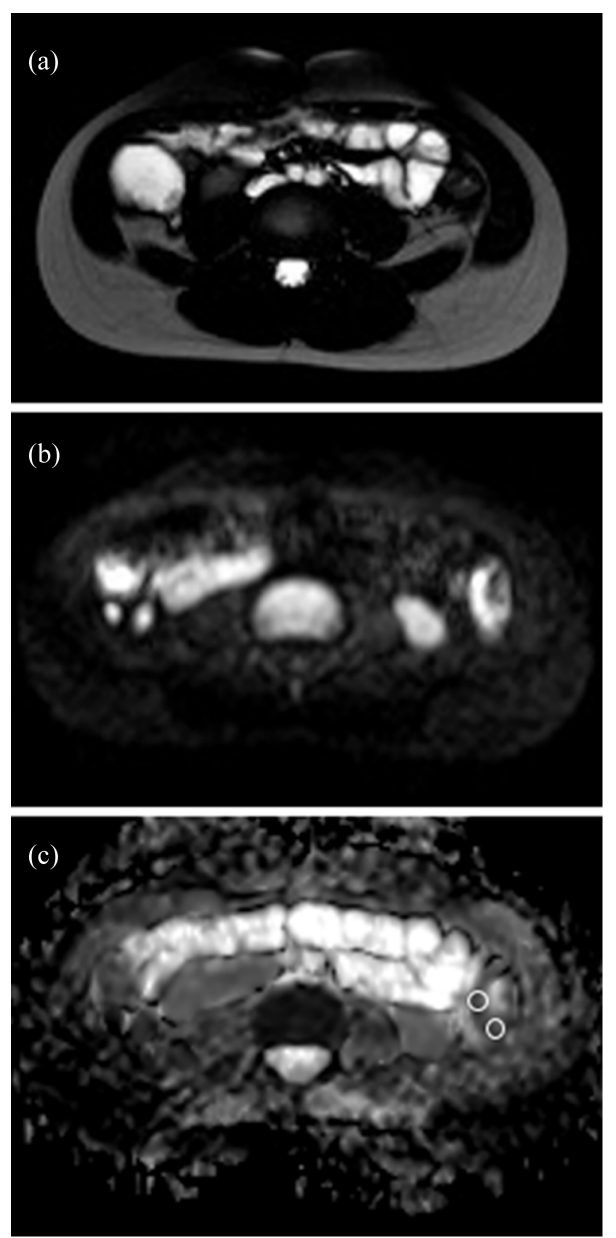

Figure 3. Ulcerative Colitis. (a) Axial balanced fast field echo (B-FFE) sequenceDiffuse bowel wall thickening of the descending colon; (b) Axial diffusion weighted (DWI) sequence-hyperintense signal at the same level; (c) Apparent diffusion coefficient (ADC) map-The ADC value was $0.817 \times 10^{-3} \mathrm{~mm}^{2} / \mathrm{s}$.

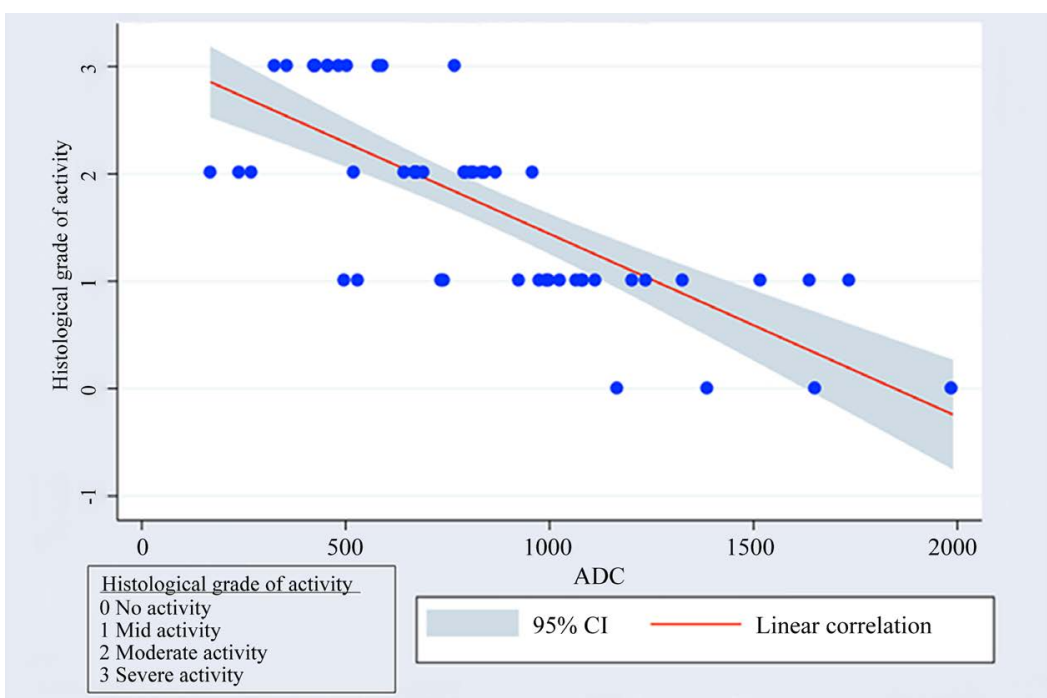

Figure 4. Linear correlation between apparent diffusion coefficient ADC values and the histologic classification of disease severity. 
Table 4. Observed, expected and Kappa agreement between histological and sonographic classification of bowel disease severity.

\begin{tabular}{|c|c|c|c|c|c|c|}
\hline & Agreement & $\begin{array}{c}\text { Expected } \\
\text { agreement }\end{array}$ & Карра & $\begin{array}{l}\text { Std. } \\
\text { Error }\end{array}$ & $\mathbf{z}$ & $\mathbf{p}$ \\
\hline $\begin{array}{c}\text { Histological vs } \\
\text { Sonographic } \\
\text { classification }\end{array}$ & 86.8 & 29.0 & 0.81 & 0.09 & 9.56 & 0.00 \\
\hline
\end{tabular}

Table 5. Sensitivity, specificity, positive and negative predicted values (with 95\% confidence interval indication) for Color Doppler Ultrasonography (CD-US) results associated to histological classification of disease severity.

\begin{tabular}{|c|c|c|c|c|}
\hline \multicolumn{5}{|c|}{ CD-US } \\
\hline & Sensitivity & Specificity & PPV & NPV \\
\hline No activity & $80(28.4-99.5)$ & $100(92.6-100.0)$ & $100(39.8-100.0)$ & 98.0 (89.1 - 99.9) \\
\hline Mild activity & 93.3 (68.1 - 99.8) & $86.8(71.9-95.6)$ & 73.7 (48.8 - 90.9) & $97.1(84.7-99.9)$ \\
\hline Moderate activity & $84.2(60.4$ - 96.6) & 94.1 (80.3 - 99.3) & $88.9(65.3$ - 98.6) & $91.4(76.9-98.2)$ \\
\hline Severe activity & 85.7 (57.2 - 98.2) & $100.0(91.1-100.0)$ & $100.0(73.5-100.0)$ & $95.1(83.5-99.4)$ \\
\hline
\end{tabular}

\section{Discussion}

Assessment of the disease activity is fundamental for a rational management of IBD. Recently, there has been a growing awareness of the shortcomings of only a clinical evaluation for assessment of the disease activity. Some patients with an established diagnosis of IBD and symptoms compatible with a disease flare do not have evidence of active IBD according to laboratory, endoscopic and radiologic criteria [23].

On the other hand, the laboratory tests and radiologic evaluation of asymptomatic affected patients can sometimes reveal a relapse [24]. In a sizable proportion of cases, IBD starts in childhood and requires numerous follow-up examinations during life: therefore, specific, noninvasive, well-tolerated and repeatable imaging techniques are needed.

According to the literature, US has been widely recognized as a first line imaging modality in children [25]-[28]. The detection of an abnormal bowel wall thickening is a common US finding on which to diagnose IBD, using different thickness values as a threshold, ranging in children from 1.5 to $3 \mathrm{~mm}$ for the terminal ileum and from 2 to $3 \mathrm{~mm}$ for the colon [29] [30], with 75/94\% sensitivity and 67/100\% specificity [31]. Vascular lesions and microvascular changes are well known features of IBD: in several studies, Doppler ultrasound has been found increasingly useful for the investigation of splanchnic hemodynamics and it has been demonstrated that the vascularization pattern can be used to determine a sonographic activity score [32]-[35].

Ruess et al. stressed that a bowel wall thickness $>3 \mathrm{~mm}$ and increased bowel wall vascularity are correlated with common laboratory and clinical parameters of disease activity in patients with Crohn's disease and ulcerative colitis [36].

Haber et al., comparing US and histology in a population of 78 children, demonstrated a statistically significant relationship between maximal bowel wall thickness and disease activity, for both Crohn's and RCU [29]. In our series we obtained similar results, with a statistically significant agreement (86.8\%).

We compared the US findings [increased bowel wall thickness (using a threshold of $3 \mathrm{~mm}$ for the terminal ileum and $2 \mathrm{~mm}$ for the colon) and intramural vascularity at the site of thickening] with intestinal biopsies in the evaluation of the grade of disease activity.

In a study done by Reimund et al. of the US diagnostic value in Crohn's disease and ulcerative colitis, the estimated sensitivity and specificity of US in the evaluation of disease activity were $94 \%$ and $67 \%$, respectively [37]; we obtained a similar sensitivity (80/93.3\%) and higher specificity (86.8/100\%).

Drews et al used the Limberg classification in a study including 32 adults, and demonstrated a significant association ( $\mathrm{p}<0.005)$ between the results of histology and bowel wall vascularity in the terminal ileum $(\mathrm{n}=0.66$; sensitivity 95\%; specificity 69\%) [20]. These findings were confirmed by Sasaki et al., who evaluated 108 Crohn's patients, showing that the Limberg score reflects the endoscopic grade of activity, particularly in moderate and severe inflammation [38]. Using the same score we obtained similar results, with a significant associa- 
tion between US and histology $(\mathrm{k}=0.81)$, comparable sensitivity $(80 / 93.3 \%)$ and higher specificity (86.8/ $100 \%)$.

In a study including 44 children, Bremner et al. proposed that an increased bowel wall thickness in the ileum and in the colon has a high positive predictive value in moderate and severe inflammation [39]. In our study we found the highest PPV (100\%) in inactive and severe inflammation, versus $73 \%$ and $88.9 \%$ in mild and moderate activity respectively.

MRE is another well established imaging modality that does not expose patients to ionizing radiation, and enables the study of IBD with conventional and functional sequences. DWI is a technique that uses the diffusion of water in biologic tissues to produce images: it provides functional, quantitative information about tissue cellularity [16] [40].

Recently, in several studies, DWI-MRE, associated with ADC measurements, has been shown to reflect an abnormal activity in CD patients [41] [42]. Specifically, intestinal inflammatory lesions are characterized by a brighter signal in the b-value images and lower ADC values in normal segments. The reduction of ADC values in abnormal segments has been suggested to reflect an increased cellularity which, if present, would restrict the diffusion of the water molecules in the tissue [43] [44].

Oto et al. evaluated 53 intestinal segments (19 pathologic and 34 controls) with DWI-MRE in 11 adult patients. ADC mean values were $1.59 \pm 0.45 \times 10^{-3} \mathrm{~mm}^{2} / \mathrm{sec}$ (range $0.46-2.50 \times 10^{-3} \mathrm{~mm}^{2} / \mathrm{sec}$ ) in cases of inflammation and $2.74 \pm 0.68 \times 10^{-3} \mathrm{~mm}^{2} / \mathrm{sec}$ (range $1.44-4.03 \times 10^{-3} \mathrm{~mm}^{2} / \mathrm{s}$ ) in normal segments [45].

Kiryu et al. measured ADC values of the small and large bowel in 31 adults and compared the results of pathologic bowel segments to the normal ones: the ADC was lower in inflamed segments than healthy ones (Small bowel: $1.61 \pm 0.44 \times 10^{-3} \mathrm{~mm}^{2} / \mathrm{sec}$ vs $2.56 \pm 0.51 \times 10^{-3} \mathrm{~mm}^{2} / \mathrm{sec}$. Large bowel: $1.52 \pm 0.43 \times 10^{-3} \mathrm{~mm}^{2} / \mathrm{sec}$ versus $2.31 \pm 0.59 \times 10^{-3} \mathrm{~mm}^{2} / \mathrm{sec}$ ) [46]. Ream et al. studied a group of pediatric patients (mean age: 14.6 years) affected by Crohn's disease. Mean ADC values of the inflamed bowel wall were $1.4 \pm 0.3 \times 10^{-3} \mathrm{~mm}^{2} / \mathrm{sec}$ [47]. In a population of 33 children, Neubauer et al retrospectively calculated ADC values of pathologic small bowel [0.84/1.40 $\times 10^{-3} \mathrm{~mm}^{2} / \mathrm{sec}$ (range $\left.\left.1.16 \pm 0.18\right)\right]$ and colon [0.84/1.60 $\left.\times 10^{-3} \mathrm{~mm}^{2} / \mathrm{sec}(1.21 \pm 0.21)\right]$ [48] .

In a recent prospective research (2014) Tielbeek et al. made a comparison between dynamic contrast enhanced MRE (DCE-MRE) and DWI-ADC in affected adults; the results were referred to histology (AIS, acute inflammation score and FS, fibrosis score). The mean ADC value was 1988 (range 1216/4198): there was a trend towards decreased ADC values being associated with AIS, but this was not significant, while a decrease in ADC values was significantly correlated with FS [49].

Our results were comparable with those of other studies involving children: mean ADC values were $0.837 \pm$ $0.4 \times 10^{-3} \mathrm{~mm}^{2} / \mathrm{sec}$ in our study, which are similar to the values previously reported by Ream $\left(1.4 \pm 0.3 \times 10^{-3}\right.$ $\left.\mathrm{mm}^{2} / \mathrm{sec}\right)$ [47] and Neubauer $\left(0.84 / 1.40 \times 10^{-3} \mathrm{~mm}^{2} / \mathrm{sec}\right.$ in the ileum and $0.84 / 1.60 \times 10^{-3} \mathrm{~mm}^{2} / \mathrm{sec}$ in the colon) [48].

As already observed by Ream et al, as compared to studies involving adults our results were slightly lower [47]. The mean ADC value was $0.837 \pm 0.4 \times 10^{-3} \mathrm{~mm}^{2} / \mathrm{sec}$ in our study vs: $1.59 \pm 0.45 \times 10^{-3} \mathrm{~mm}^{2} / \mathrm{sec}$ in Oto's study [45], $1.61 \pm 0.44 \times 10^{-3} \mathrm{~mm}^{2} / \mathrm{sec}$ and $1.52 \pm 0.43 \times 10^{-3} \mathrm{~mm}^{2} / \mathrm{sec}$ in the ileum and in the colon, respectively, in Kyriu's study [46] and $1988\left(1988 \times 10^{-3}\right)$ in Tielbeek's study [49].

In our experience the ADC values were negatively correlated to the disease severity and, assuming $1 \times 10^{-3}$ $\mathrm{mm}^{2} / \mathrm{sec}$ as a cut off value, were useful to distinguish between inactive and moderate-severe active disease in 83.3\% cases.

This study has some limitations. Firstly, the sample size is relatively small: this is partially due to the inclusion criterion that all patients included must have undergone US, MRI and intestinal biopsy during the same week.

Secondly, as the study is retrospective, we were limited to only two b-values ( 0 and $600 \mathrm{~mm}^{2} / \mathrm{sec}$ ) (b-value measures the degree of the diffusion weighting applied. It indicates the amplitude $(\mathrm{G})$, the time of applied gradients $(\delta)$ and the duration between the paired gradients $(\Delta)$. It is calculated as: $b=\gamma^{2} \mathrm{G}^{2} \delta^{2}(\Delta-\delta / 3)$ that were used for our routine DWI examinations; however, these b-values are similar to those described in previous studies [45] [47] [50] assessing DWI in children or adults.

Thirdly, the precise placement of ROIs on the ADC map is very challenging.

\section{Conclusion}

CD-US and DWI are useful in the evaluation of IBD severity and can have a particular value in the follow-up in 
childhood. Both techniques could be employed to identify disease flares, even in asymptomatic patients, to monitor the treatment and to evaluate surgical recurrence.

\section{Acknowledgements}

We would like to express our great appreciation to our TSRM Mr. Salvatore De Ceglie and Mr. Vito Savino, for their contribution to the collection of MR images and to Dr. Babette Pragnell for her precious help in the correction of written English.

\section{References}

[1] Gaya, D.R., Russell, R.K. and Nimmo, E.R. (2006) New Genes in Inflammatory Bowel Disease: Lessons for Complex Diseases? The Lancet, 367, 1271-1284. http://dx.doi.org/10.1016/S0140-6736(06)68345-1

[2] IBD Working Group of the European Society of Paediatric Gastroenterology, Hepatology and Nutrition (ESPGHAN) (2005) Inflammatory Bowel Disease in Children and Adolescents: Recommendations for Diagnosis—The Porto Criteria. Journal of Pediatric Gastroenterology \& Nutrition, 41, 1-7. http://dx.doi.org/10.1097/01.MPG.0000163736.30261.82

[3] Silverberg, M.S., Satsangi, J., Ahmad, T., et al. (2005) Toward an Integrated Clinical, Molecular and Serological Classification of Inflammatory Bowel Disease: Report of a Working Party of the 2005 Montreal World Congress of Gastroenterology. Canadian Journal of Gastroenterology, 19, 5-36.

[4] Benchimol, E.I., Guttmann, A., Griffiths, A.M., et al. (2009) Increasing Incidence of Paediatric Inflammatory Bowel Disease in Ontario, Canada: Evidence from Health Administrative Data. Gut, 58, 1490-1497. http://dx.doi.org/10.1136/gut.2009.188383

[5] Yong Hoon, K. and Yong Joo, K. (2013) Pre-Diagnostic Clinical Presentations and Medical History Prior to the Diagnosis of Inflammatory Bowel Disease in Children. Pediatric Gastroenterology, Hepatology and Nutrition, 16, 178-184. http://dx.doi.org/10.5223/pghn.2013.16.3.178

[6] Molodecky, N.A., Shian Soon, I., Rabi, D.M., et al. (2012) Increasing Incidence and Prevalence of the Inflammatory Bowel Diseases with Time, Based On Systematic Review. Gastroenterology, 142, 46-54. http://dx.doi.org/10.1053/j.gastro.2011.10.001

[7] Sandhu, B.K., Fell, J.M., Beattie, R.M., et al. (2010) Guidelines for the Management of Inflammatory Bowel Disease (IBD) in Children in the United Kingdom. Journal of Pediatric Gastroenterology \& Nutrition, 50, S1-S13. http://dx.doi.org/10.1097/MPG.0b013e3181c92c53

[8] Paolantonio, P., Ferrari, R. and Vecchietti, F. (2009) Current Status of MR Imaging in the Evaluation of IBD in a Pediatric Population of Patients. European Journal of Radiology, 69, 418-424. http://dx.doi.org/10.1016/j.ejrad.2008.11.023

[9] Dubinsky, M. (2008) Special Issues in Pediatric Inflammatory Bowel Disease. World Journal of Gastroenterology, 14, 413-420. http://dx.doi.org/10.3748/wjg.14.413

[10] Athanasakos, A., Mazioti, A., Economopoulos, N., et al. (2015) Inflammatory Bowel Disease-The Role of CrossSectional Imaging Techniques in the Investigation of the Small Bowel. Insights into Imaging, 6, 73-83. http://dx.doi.org/10.1007/s13244-014-0377-6

[11] Strauss, K.J. and Kaste, S.C. (2006) The ALARA (as Low as Reasonably Achievable) Concept in Pediatric Interventional and Fluoroscopic Imaging: Striving to Keep Radiation Doses as Low as Possible during Fluoroscopy of Pediatric Patients-A White Paper Executive. Pediatric Radiology, 36, 110-112. http://dx.doi.org/10.1007/s00247-006-0184-4

[12] Dambha, F., Tanner, J., Carroll, N., et al. (2014) Diagnostic Imaging in Crohn's Disease: What Is the New Gold Standard? Best Practice \& Research Clinical Gastroenterology, 28, 421-436. http://dx.doi.org/10.1016/j.bpg.2014.04.010

[13] Asthana, A.K., Friedman, A.B., Maconi, G., et al. (2015) The Failure of Gastroenterologists to Apply Intestinal Ultrasound in Inflammatory Bowel Disease in the Asia-Pacific: A Need for Action. Journal of Gastroenterology and Hepatology, 30, 446-452. http://dx.doi.org/10.1111/jgh.12871

[14] Buisson, A., Joubert, A., Montoriol, P.F., et al. (2013) Diffusion-Weighted Magnetic Resonance Imaging for Detecting and Assessing Ileal Inflammation in Crohn's Disease. Alimentary Pharmacology \& Therapeutics, 37, 537-545. http://dx.doi.org/10.1111/apt.12201

[15] Benitez, J.M., Meuwis, M.A., Reenaers, C., et al. (2015) Role of Endoscopy, Cross-Sectional Imaging and Biomarkers in Crohn's Disease Monitoring. Gut, 62, 1806-1816. http://dx.doi.org/10.1136/gutjnl-2012-303957

[16] Sinha, R., Rajiah, P., Ramachandran, I., et al. (2013) Diffusion-Weighted MR Imaging of the Gastrointestinal Tract: Technique, Indications, and Imaging Findings. Radiographics, 33, 655-676. http://dx.doi.org/10.1148/rg.333125042 
[17] Taylor, S., Mallett, S. and Bhatnagar, G. (2014) METRIC (MREnterography or ulTRasound in Crohn’s Disease): A Study Protocol for a Multicentre, Non-Randomised, Single-Arm, Prospective Comparison Study of Magnetic Resonance Enterography and Small Bowel Ultrasound Compared to a Reference Standard in Those Aged 16 and Over. BMC Gastroenterology, 14, 142. http://dx.doi.org/10.1186/1471-230X-14-142

[18] Estay, C., Simian, D., Lubascher, J., et al. (2015) Ionizing Radiation Exposure in Patients with Inflammatory Bowel Disease: Are We Overexposing Our Patients? Journal of Digestive Diseases, 16, 83-89. http://dx.doi.org/10.1111/1751-2980.12213

[19] Limberg, B. (1999) Diagnosis of Chronic Inflammatory Bowel Disease by Ultrasonography. Zeitschrift für Gastroenterologie, 37, 495-508.

[20] Drews, B.H., Barth, T.F.E., Hänle, M.M., et al. (2009) Comparison of Sonographically Measured Bowel Wall Vascularity, Histology and Disease Activity in Crohn’s Disease. European Radiology, 19, 1379-1386. http://dx.doi.org/10.1007/s00330-008-1290-5

[21] Villanacci, V., Antonelli, E., Geboes, K., et al. (2013) Histological Healing in Inflammatory Bowel Disease: A Still Unfulfilled Promise. World Journal of Gastroenterology, 19, 968-978. http://dx.doi.org/10.3748/wjg.v19.i7.968

[22] Geboes, K., Riddell, R., Öst, A., et al. (2000) A Reproducible Grading Scale for Histological Assessment of Inflammation in Ulcerative Colitis. Gut, 47, 404-409. http://dx.doi.org/10.1136/gut.47.3.404

[23] Panes, J., Bouzas, R., Chaparro, M., et al. (2011) Systematic Review: The Use of Ultrasonography, Computed Tomography and Magnetic Resonance Imaging for the Diagnosis, Assessment of Activity and Abdominal Complications of Crohn's Disease. Alimentary Pharmacology \& Therapeutics, 34, 125-145. http://dx.doi.org/10.1111/j.1365-2036.2011.04710.x

[24] Pariente, B., Cosnes, J., Danese, S., et al. (2011) Development of the Crohn's Disease Digestive Damage Score, the Lemann Score. Inflammatory Bowel Diseases, 17, 1415-1422. http://dx.doi.org/10.1002/ibd.21506

[25] Alison, M., Kheniche, A., Azoulay, R., et al. (2007) Ultrasonography of Crohn Disease in Children. Pediatric Radiology, 37, 1071-1082. http://dx.doi.org/10.1007/s00247-007-0559-1

[26] Strobel, D., Goertz, R.S. and Bernatik, T. (2011) Diagnostics in Inflammatory Bowel Disease: Ultrasound. World Journal of Gastroenterology, 17, 3192-3197.

[27] Parente, F., Greco, S., Molteni, M., et al. (2004) Modern Imaging of Crohn's Disease Using Bowel Ultrasound. Inflammatory Bowel Diseases, 10, 452-461. http://dx.doi.org/10.1097/00054725-200407000-00022

[28] Mackalaski, B.A. and Bernstein, C.N. (2006) New Diagnostic Imaging Tools for Inflammatory Bowel Disease. Gut, 55, 733-741. http://dx.doi.org/10.1136/gut.2005.076612

[29] Haber, H.P., Busch, A., Ziebach, R., et al. (2002) Ultrasonographic Findings Correspond to Clinical, Endoscopic and Histologic Findings in Inflammatory Bowel Disease and Other Enterocolitides. Journal of Ultrasound in Medicine, 21, 375-382.

[30] Canani, R.B., De Horatio, L.T., Terrin, G., et al. (2006) Combined Use of Noninvasive Tests Is Useful in the Initial Diagnosis Approach to a Child with Suspected Inflammatory Bowel Disease. Journal of Pediatric Gastroenterology and Nutrition, 42, 9-15. http://dx.doi.org/10.1097/01.mpg.0000187818.76954.9a

[31] Panes, J., Bouhnik, Y., Reinisch, W., et al. (2013) Imaging Techniques for Assessment of Inflammatory Bowel Disease: Joint ECCO and ESGAR Evidence-Based Consensus Guidelines. Journal of Crohn's and Colitis, 7, 556-585. http://dx.doi.org/10.1016/j.crohns.2013.02.020

[32] Maconi, G., Parente, F., Bollani, S., et al. (1998) Factors Affecting Splanchnic Hemodynamics in Crohn’s Disease: A Prospective Controlled Study Using Doppler Ultrasound. Gut, 43, 645-650. http://dx.doi.org/10.1136/gut.43.5.645

[33] Spalinger, J., Patriquin, H. and Miron, M.C. (2000) Doppler US in Patients with Crohn Disease: Vessel Density in the Diseased Bowel Reflects Disease Activity. Radiology, 217, 787-791. http://dx.doi.org/10.1148/radiology.217.3.r00dc19787

[34] Yekeler, E., Danalioglu, A. and Movasseghi, B. (2005) Crohn Disease Activity Evaluated by Doppler Ultrasonography of the Superior Mesenteric Artery and the Affected Small Bowel Segments. Journal of Ultrasound in Medicine, 24, 5965.

[35] Neye, H., Voderholzer, W. and Rickes, S. (2004) Evaluation of Criteria for the Activity of Crohn's Disease by Power Doppler Sonography. Digital Distribution, 22, 67-72. http://dx.doi.org/10.1159/000078737

[36] Ruess, L., Blask, A.R. and Bulas, D.I. (2000) Inflammatory Bowel Disease in Children and Young Adults: Correlation of Sonographic and Clinical Parameters during Treatment. American Journal of Roentgenology, 175, 79-84. http://dx.doi.org/10.2214/ajr.175.1.1750079

[37] Reimund, J.M., Jung Chaigneau, E. and Chamouard, P. (1999) Diagnostic Value of High Resolution Sonography in Crohn's Disease and Ulcerative Colitis. Gastroentérologie Clinique et Biologique, 23, 740-746. 
[38] Sasaki, T., Kunisaki, R. and Kinoshita, H. (2014) Use of Color Doppler Ultrasonography for Evaluating Vascularity of Small Intestinal Lesions in Crohn's Disease: Correlation with Endoscopic and Surgical Macroscopic Findings. Scandinavian Journal of Gastroenterology, 49, 295-301. http://dx.doi.org/10.3109/00365521.2013.871744

[39] Bremner, A.R., Griffiths, M. and Argent, J.D. (2006) Sonographic Evaluation of Inflammatory Bowel Disease: A Prospective Blinded Comparative Study. Pediatric Radiology, 36, 947-953. http://dx.doi.org/10.1007/s00247-006-0245-8

[40] Oussalah, A., Laurent, V., Bruot, O., et al. (2010) Diffusion-Weighted Magnetic Resonance without Bowel Preparation for Detecting Colonic Inflammation in Inflammatory Bowel Disease. Gut, 59, 1056-1065. http://dx.doi.org/10.1136/gut.2009.197665

[41] Kinner, S., Blex, S., Maderwald, S., et al. (2014) Addition of Diffusion-Weighted Imaging Can Improve Diagnostic Confidence in Bowel MRI. Clinical Radiology, 69, 372-377. http://dx.doi.org/10.1016/j.crad.2013.09.022

[42] Sakuraba, H., Ishiguro, Y., Hasui, K., et al. (2014) Prediction of Maintained Mucosal Healing in Patients with Crohn’s Disease under Treatment with Infliximab Using Diffusion-Weighted Magnetic Resonance Imaging. Digestion, 89, 4954. http://dx.doi.org/10.1159/000356220

[43] Freiman, M., Perez-Rossello, J.M. and Callahan, M.J. (2013) Characterization of Fast and Slow Diffusion from Diffusion-Weighted MRI of Pediatric Crohn's Disease. Journal of Magnetic Resonance Imaging, 37, 156-163. http://dx.doi.org/10.1002/jmri.23781

[44] Griffin, N., Grant, L.A. and Anderson, S. (2012) Small Bowel MR Enterography: Problem Solving in Crohn's Disease. Insights Imaging, 3, 251-263. http://dx.doi.org/10.1007/s13244-012-0154-3

[45] Oto, A., Zhu, F., Kulkarni, K., et al. (2009) Evaluation of Diffusion-Weighted MR Imaging for Detection of Bowel Inflammation in Patients with Crohn's Disease. Academic Radiology, 16, 597-603. http://dx.doi.org/10.1016/j.acra.2008.11.009

[46] Kiryu, S., Dodanuki, K., Takao, H., et al. (2009) Free-Breathing Diffusion Weighted Imaging for the Assessment of Inflammatory Activity in Crohn's Disease. Journal of Magnetic Resonance Imaging, 29, 880-886. http://dx.doi.org/10.1002/jmri.21725

[47] Ream, J.M., Dillman, J.R., Adler, J., et al. (2013) MRI Diffusion-Weighted Imaging (DWI) in Pediatric Small Bowel Crohn Disease: Correlation with MRI Findings of Active Bowel Wall Inflammation. Pediatric Radiology, 43, 10771085. http://dx.doi.org/10.1007/s00247-013-2712-3

[48] Neubauer, H., Pabst, T. and Dick, A. (2013) Small Bowel MRI in Children and Young Adults with Crohn Disease: Retrospective Head-to-Head Comparison of Contrast-Enhanced and Diffusion-Weighted MRI. Pediatric Radiology, 43, 103-114. http://dx.doi.org/10.1007/s00247-012-2492-1

[49] Tielbeek, J.A., Ziech, M.L., Li, Z., et al. (2014) Evaluation of Conventional, Dynamic Contrast Enhanced and Diffusion Weighted MRI for Quantitative Crohn's Disease Assessment with Histopathology of Surgical Specimens. European Radiology, 24, 619-629. http://dx.doi.org/10.1007/s00330-013-3015-7

[50] Oto, A., Kayhan, A. and Williams, J.T.B. (2011) Active Crohn’s Disease in the Small Bowel: Evaluation by Diffusion Weighted Imaging and Quantitative Dynamic Contrast Enhanced MR Imaging. Journal of Magnetic Resonance Imaging, 33, 615-624. http://dx.doi.org/10.1002/jmri.22435 